\title{
Estrutura dos meios de hospedagem para pessoas com deficiência em Santa Catarina
}

Suzana Rodrigues Macedo. Mestre em Meteorologia. Faculdade de Tecnologia Senac Florianópolis - Brasil. suzana@prof.sc.senac.br Carlos André de Sousa Rocha. Mestre em Ciências da Computação. Faculdade de Tecnologia Senac Criciúma - Brasil. carlos.rocha@prof.sc.senac.br

\section{RESUMO}

O presente trabalho teve como objetivo, realizar uma análise preliminar em relação à acessibilidade para pessoas com deficiência nos meios de hospedagens de Santa Catarina. Justifica-se a importância da pesquisa diante da tendência de mercado na adaptação do setor turístico para atender às demandas das pessoas com deficiência e ressaltando a Lei Federal 13.146 de 06 de julho de 2015, que prevê que os setores públicos e privados criem condições para acessibilidade. A metodologia aplicada na pesquisa foi exploratória e descritiva, a partir de um levantamento do número de meios de hospedagens existentes em Santa Catarina registrados no CADASTUR e verificação de sua estrutura informada no cadastro, pautada na NBR 9050, sondando a respeito da adaptação das unidades habitacionais dos meios de hospedagens para a acessibilidade de pessoas com deficiência. A pesquisa mostra que é pequena a parcela de unidades habitacionais com algum tipo de adaptação para acessibilidade, demonstrando uma necessidade de adequação por parte dos meios de hospedagens em Santa catarina, para atender a esta demanda.

Palavras-chave: Acessibilidade. Hotéis. Turismo Santa Catarina.

\section{Structure of accommodation facilities for people with special needs in Santa Catarina}

\begin{abstract}
This research aimed to conduct a preliminary analysis in relation to accessibility for people with special needs in the accommodation facilities of Santa Catarina. The importance of the research is justified due to the need of the market to adapt the tourism sector to the demands of people with special needs as well as to conform to the Federal Law 13146 of July 6,2015 , which provides that the public and the private sectors create conditions for accessibility. The methodology used in the research was exploratory and descriptive having as a starting point a survey of the number of existing accommodation facilities in Santa Catarina recorded in CADASTUR and the verification of their structure according to reported in the register, based on the NBR 9050, verifying the adaptation of housing units in those the means of accommodation in what concerns accessibility for people with special needs. This research shows that the number of housing units with some kind of adaptation to facilitate accessibility for people with special needs is low, demonstrating the need for means of accommodation in Santa Catarina to adequate in order to meet this demand.
\end{abstract}

Keywords: Accessibility. Hotels. Tourism Santa Catarina. 


\section{INTRODUÇÃO}

O Estado de Santa Catarina é reconhecido no Brasil e no exterior por seus atrativos turísticos, principalmente devido a sua natureza exuberante localizada em seu litoral. O turismo na região conta com um litoral que abriga praias, fontes de águas termais, dunas, cânions, serras e campos naturais; constituindo, assim, uma variedade de diferentes paisagens que servem para o desenvolvimento de diferentes tipos de turismo.

Por suas especificidades regionais, a atividade turística constitui uma importante fonte econômica para o Estado, concentrando-se na alta estação de veraneio a maior demanda turística de lazer que, ao longo dos anos, contribuiu para a consolidação progressiva de uma estrutura turística, reforçando a presença de Santa Catarina no cenário brasileiro dos destinos turísticos mais procurados do país.

Os meios de hospedagens encontrados no Estado são diversificados e com o maior número de estabelecimentos associados ao turismo de lazer concentrado na faixa litorânea, próximos às praias. As regiões de Imbituba, Porto Belo e o Sul da llha de Santa Catarina são as que apresentam maior número de Pousadas. Já nas cidades de Balneário Camboriú e Florianópolis concentram-se os hotéis de lazer e os três maiores resorts do Estado.

Em relação à estrutura, em 2011, a ABIH-SC lançou o Guia de Hotéis de Santa Catarina, que trazia a informação da existência de 2.500 meios de hospedagens, contando com 60 mil quartos e 160 mil leitos para o acolhimento dos turistas.

Em meio ao turismo convencional, surge como oportunidade de negócios o turismo adaptado: opções adaptadas para oferecer acessibilidade a pessoas com deficiência, promovendo a inclusão destas nas atividades turísticas.

Com base em dados da Organização das Nações Unidas (ONU), cerca de 10\% da população mundial aproximadamente 650 milhões de pessoas, vivem com algum tipo de deficiência e dessas, $80 \%$ vivem em países em desenvolvimento (ONU, 2016). No Brasil, de acordo com o levantamento do Instituto Brasileiro de Geografia e Estatística (IBGE, 2011), 6,2\% da população brasileira são portadoras de pelo menos um tipo de deficiência, sendo 3,6\% com deficiência visual, destacandose a Região Sul com a maior proporção de pessoas com deficiência visual (5,4\%). A pesquisa mostra ainda que $6,6 \%$ dos brasileiros usam algum recurso para auxiliar a locomoção, como bengala articulada ou cão-guia.

A FECOMÉRCIO (2016) chama atenção para o crescimento deste mercado, citando o exemplo de dois hotéis fazenda, Parque dos Sonhos e Campo dos Sonhos, localizados em Minas Gerais e em São Paulo, respectivamente. Ambos são meios de hospedagens que investiram no atendimento a pessoas com deficiências, aumentando a taxa de ocupação em 50\%.

Com base nesse cenário, o problema da pesquisa consistiu em investigar se os meios de hospedagens no Estado de Santa Catarina cadastrados no CADASTUR possuem estrutura para receber turistas com deficiência em suas unidades habitacionais. O CADASTUR é o Sistema de Cadastro de pessoas físicas e jurídicas que atuam no setor do turismo. Este sistema é executado pelo Ministério do Turismo, em parceria com os Órgãos Oficiais de Turismo nos 26 Estados do Brasil e no Distrito Federal.

O objetivo geral deste trabalho é promover a reflexão sobre a estrutura dos meios de hospedagens localizados em Santa Catarina, com relação às unidades habitacionais adaptadas a pessoas com deficiência: cadeirantes, utilizadores de cão-guia como recurso de apoio à locomoção, deficientes auditivos . 
Os objetivos específicos são:

- Verificar a quantidade de meios de hospedagens existentes em Santa Catarina, com cadastro regular no CADASTUR;

- Identificar quantos meios de hospedagens cadastrados possuem unidades habitacionais adaptadas para pessoas com deficiência (quanto à acessibilidade para cadeirantes, existência de serviços especiais para deficientes auditivos e acessibilidade de pessoas dependentes de cão-guia para locomoção).

\section{REVISÃO TEÓRICA}

O turismo, segundo Fuster (1999, p. 11), "é, de um lado, conjunto de turistas, de outro, os fenômenos e as relações que essa massa produz em consequência de suas viagens". Trigo (1999, p. 12) define o turismo como:

atividade humana intencional que serve como meio de comunicação e como elo de interação entre povos, tanto dentro como fora de um país. Envolve o deslocamento temporário de pessoas para outras regiões ou países visando a satisfação de outras necessidades que não a de atividades remuneradas.

O turismo sustentável ajuda na preservação do meio ambiente, melhorando as condições de vida da população e uma das ações sociais que ele pode desempenhar é ser adaptado para a recepção de hóspedes com deficiência. Ruschmann (2001, p. 70) afirma que o turismo é sustentável quando visto como "a perfeita triangulação entre as destinações (seus hábitos e habitantes), os turistas e os prestadores de facilidades para os visitantes".

A Organização das Nações Unidas (ONU, 2016) destaca a necessidade de que sejam dadas às pessoas deficientes as mesmas oportunidades que os demais cidadãos de desfrutar da dinâmica econômica e social. Assim, viagens para destinos turísticos tornam-se uma forma de inclusão social. Além de promover a inclusão social, a adaptação dos meios de hospedagens pode ser uma forma de atrair um novo público-alvo para este tipo de estabelecimento. Um meio de hospedagem adaptado para receber hóspedes com deficiência, além de contar com este público, pode receber um aumento no número de hóspedes pois, além do hóspede com deficiência, possivelmente receberá também seus acompanhantes, familiares e amigos gerando um efeito multiplicador no número de vagas ocupadas. É importante considerar que as ações planejadas para adaptação de um meio de hospedagem aos hóspedes com deficiência, também poderá auxiliar às pessoas com mobilidade reduzida, como gestantes e idosos.

A norma da ABNT NBR 9050 (ABNT, 2004), especifica que em hotéis, motéis, pousadas e similares, os auditórios, salas de convenções, salas de ginástica, piscinas, entre outros, devem ser acessíveis. Além disso, 5\% dos dormitórios com sanitários, devem ser adaptados para pessoas com deficiência (sendo que deve contar no mínimo um dormitório com sanitário acessível). A norma recomenda ainda que estes dormitórios não fiquem isolados dos demais, mas distribuídos em toda a edificação, por todos os níveis de serviços e localizados em rota acessível. Recomenda-se, além disso, que outros $10 \%$ do total de dormitórios sejam adaptáveis para acessibilidade.

A exigência da unidade habitacional (apartamento com banheiro privativo) adaptada para pessoas portadoras de deficiência foi instituída a partir de 1987, para a classificação de meios de hospedagem. Os estabelecimentos construídos anteriormente têm que dispor de facilidades e adaptações mesmo que não impliquem em reforma estrutural da edificação. (EMBRATUR, 1997, p. 23). 
O Decreto 5296/04 define os meios de hospedagens como edificações de uso coletivo e dispõe sobre as condições de acessibilidade que este tipo de estabelecimento deve apresentar (BRASIL, 2004).

Em Santa Catarina, alguns estudos apontam para a baixa adesão dos meios de hospedagens ao mercado do turismo adaptado. Ely e Silva (2009) afirmam que é preciso melhorar com urgência as condições de acessibilidade dos hotéis residenciais em Florianópolis e consideraram as adaptações já realizadas, ineficientes. As autoras destacaram ainda que a acessibilidade espacial, no caso dos hotéis, não vigora como prioridade e a falta fiscalização junto aos estabelecimentos hoteleiros é precária, não atendendo à NBR 9050/2004, norma que regulamenta as condições de acessibilidade.

Uma pesquisa sobre serviços de hospedagens do IBGE (2011), aponta que em Florianópolis haviam 5440 unidades habitacionais em hotéis, sendo 53 unidades adaptadas e 888 unidades habitacionais em pousadas, com somente 20 adaptadas.

A demanda por este tipo de turismo resultou no $1^{\circ}$ Seminário Catarinense de Turismo Acessível, evento realizado em 2010, no Centrotur/ASSESC, que sintetizava o interesse do setor turístico da região pelo potencial que nele pode ser desenvolvido.

\section{PROCEDIMENTOS METODOLÓGICOS}

Inicialmente buscou-se informações junto ao Sistema de Cadastro de Pessoas Físicas e Jurídicas que atuam no Setor do Turismo (CADASTUR), a quantidade e tipos de meios de hospedagens cadastrados em Santa Catarina. Consideram-se estes dados confiáveis, visto que o mesmo é executado pelo Ministério do Turismo, em parceria com os Órgãos Oficiais de Turismo nos 26 Estados do Brasil e no Distrito Federal. A coleta de dados foi realizada em novembro de 2015.

Obtemos uma listagem com 352 meios de hospedagem, regularizados em SC, entre hotéis, pousadas, resorts, flats ou apart-hotéis e cama e café localizados em 55 cidades do Estado. Após este levantamento, foi feito contato com os meios de hospedagens para verificar quais tinham condições de acessibilidade. A Figura 1 apresenta as dez cidades com maior quantidade de meios de hospedagem. Juntas, representam mais que o dobro da quantidade de meios de hospedageem existentes nas outras 45 cidades envolvidas na pesquisa. Podemos perceber que a capital, Florianópolis, é a cidade com maior representatividade no setor, seguida por Balneário Camboriú e Bombinhas. 
Figura 1 - Frequências relativas dos Meios de Hospedagens em SC

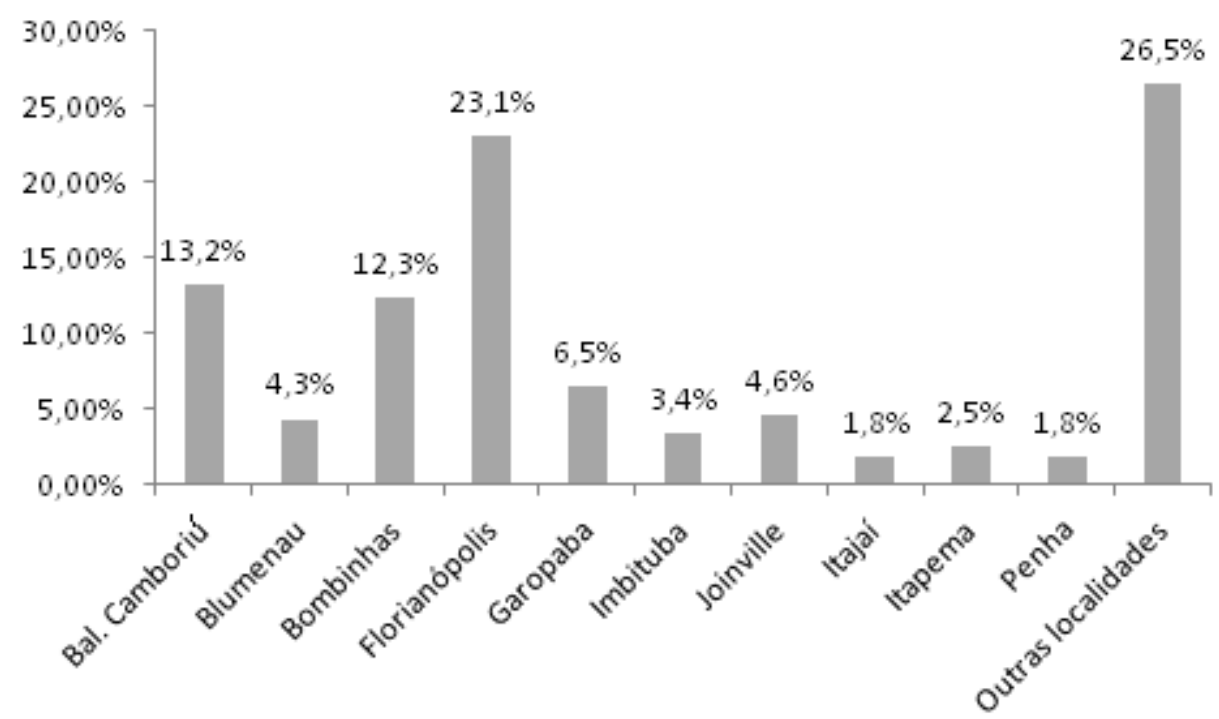

Fonte: elaborada pelos autores (2016).

\subsection{Caracterização da pesquisa}

A metodologia abordada nesta pesquisa foi qualiquantitativa, caracterizando-se como exploratória e descritiva, uma vez que se vale de dados estatísticos para a análise a partir base de dados do CADASTUR.

Pode-se considerar também a natureza bibliográfica da pesquisa, uma vez que esta traz uma investigação sobre o tema em documentos, legislações e artigos.

A coleta de dados foi realizada em novembro de 2015, considerando os registros do CADASTUR, que conta com uma lista de meios de hospedagens (hotéis, pousadas, resorts, flats ou apart-hotéis e cama e café) com registro em Santa Catarina. Com base na planilha de dados originada a partir destes registros, foram investigadas sobre a existência ou não de adaptações para pessoas portadoras de necessidades especiais, sendo especificamente: adaptações para cadeirantes, para deficientes visuais acompanhados de cão-guia, sobre adaptação de serviços para deficientes auditivos.

Para a análise dos dados, foram estudadas as frequências relativas destes, a fim de caracterizar a presença de acessibilidade nos meios de hospedagens da região.

\subsection{Análise dos Dados e Resultados}

Analisando os meios de hospedagens segundo sua caracterização: hotel, resort, cama e café, flat ou apart-hotel e pousadas - Percebemos que, conforme apresenta a Figura 2, a predominância de hotéis e, respectivamente, as pousadas. 
Figura 2 - Tipos de meios de hospedagens registrados no CADASTUR em SC.

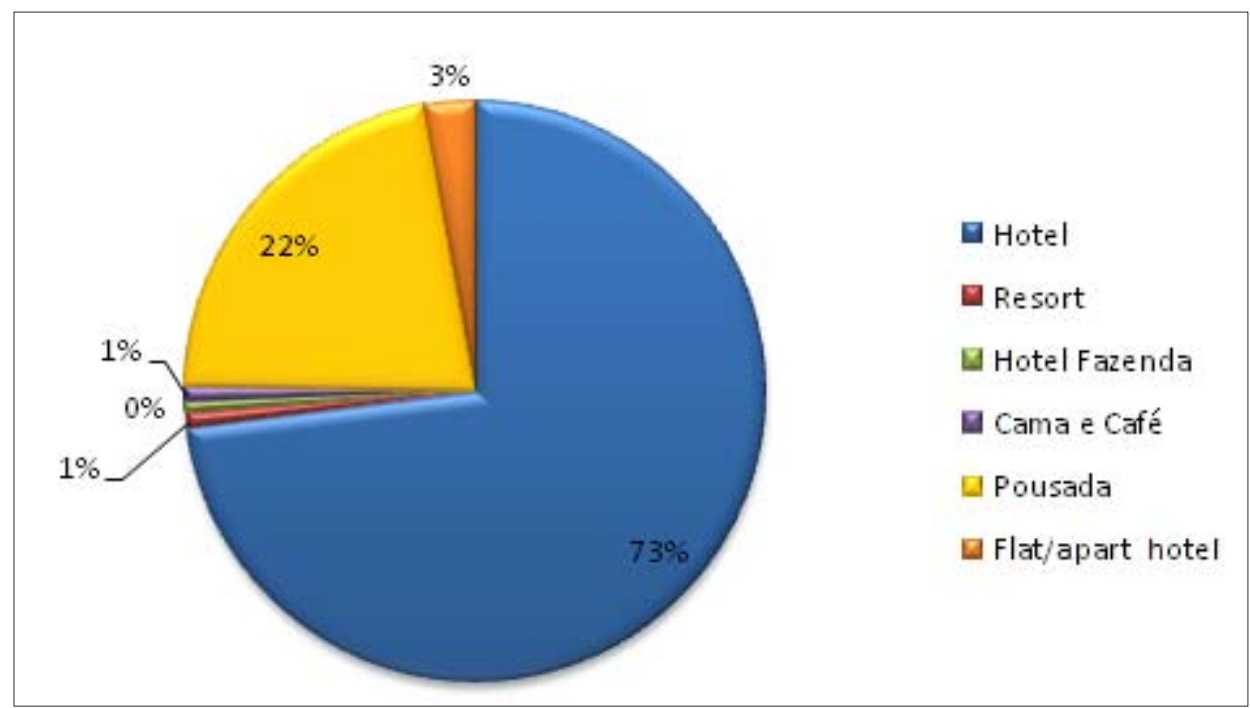

Fonte: elaborada pelos autores (2016).

Apuramos que o total de meios de hospedagens resulta na disponibilidade de 18.361 unidades habitacionais e 41.731 leitos em SC.

Com esses dados, calculamos a proporção existente entre a quantidade de leitos por unidade habitacional e por tipo de meio de hospedagem (Tabela 1). Utilizamos esta proporção para estimar a quantidade de leitos com algum tipo de adaptação, existentes nestes meios de hospedagens. Assim, estimamos que exista uma estrutura de aproximadamente 3.958 leitos com algum tipo de adaptação para a acessibilidade em SC (Tabela 1). A maioria das unidades habitacionais - com ou sem adaptações - encontram-se em hotéis (Figuras 3 e 4).

Tabela 1 - Distribuição de UH e leitos por meio de hospedagem em SC.

\begin{tabular}{cccccc}
\hline $\begin{array}{c}\text { Tipo Meio } \\
\text { Hospedagem }\end{array}$ & $\begin{array}{c}\text { Total } \\
\text { UHs }\end{array}$ & $\mathbf{N}^{\mathbf{0}}$ de leitos & $\begin{array}{c}\text { Proporção } \\
\text { leitos/UH }\end{array}$ & UHs adaptadas & $\begin{array}{c}\text { Estimativa do } \mathbf{n}^{\mathbf{0}} \\
\text { de leitos com } \\
\text { adaptações }\end{array}$ \\
\hline Hotel & 16286 & 36341 & 2,2 & 1550 & 3459 \\
Resort & 386 & 604 & 1,6 & 14 & 22 \\
Hotel Fazenda & 134 & 401 & 3,0 & 10 & 30 \\
Cama e Café & 47 & 238 & 5,1 & 1 & 5 \\
Pousada & 1149 & 3145 & 2,7 & 132 & 361 \\
Flat/Apart-hotel & 359 & 1002 & 2,8 & 29 & 81 \\
\hline Total & $\mathbf{1 8 . 3 6 1}$ & $\mathbf{4 1 . 7 3 1}$ & & $\mathbf{1 . 7 3 6}$ & $\mathbf{3 . 9 5 8}$ \\
\hline
\end{tabular}

Fonte: elaborada pelos autores (2016). 
Figura 3 - Totais de UH por tipo de meio de hospedagem em SC.

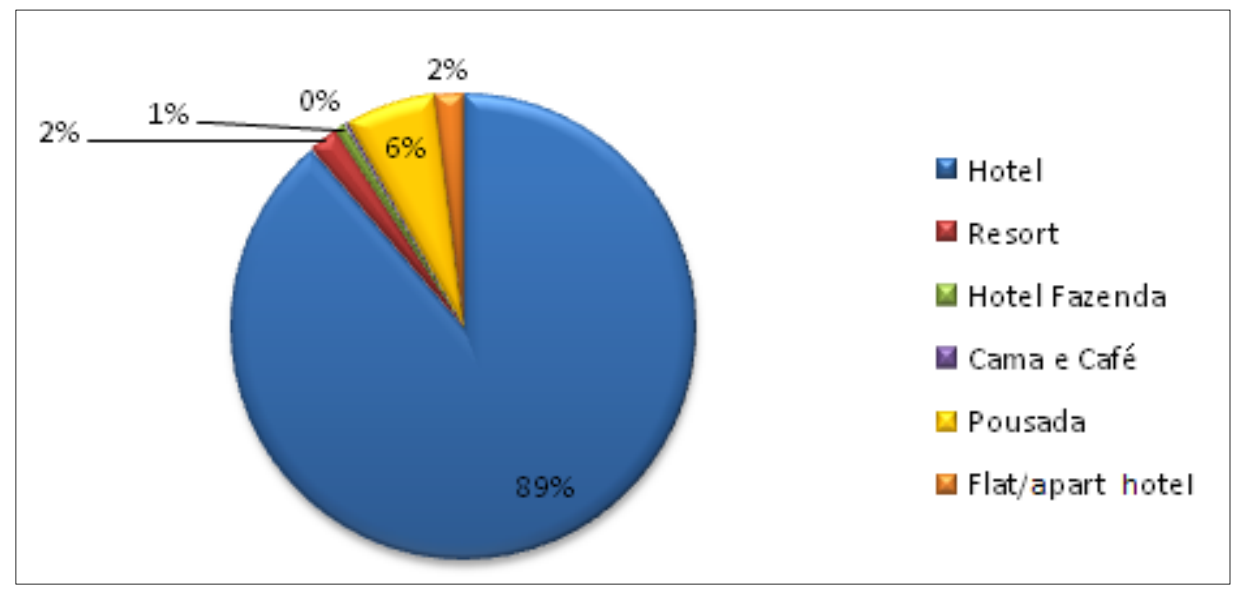

Fonte: elaborada pelos autores (2016).

Figura 4 - Distribuição das UH com adaptações por meio de hospedagem em SC.

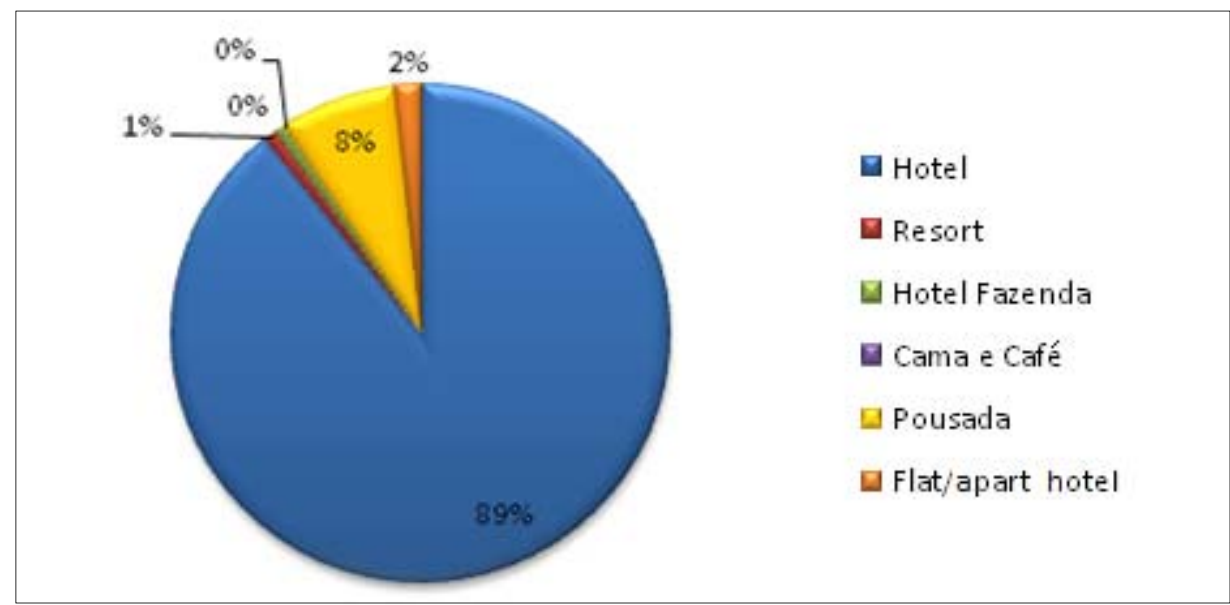

Fonte: elaborada pelos autores (2016).

A Tabela 2 resume a distribuição das unidades com algum tipo de adaptação para pessoas portadoras de necessidades especiais, por tipo de meio de hospedagem em SC.

Tabela 2 - Percentuais de UH adaptadas em SC por tipo de meio de hospedagem.

\begin{tabular}{cc}
\hline $\begin{array}{c}\text { Tipos de meios de } \\
\text { hospedagens }\end{array}$ & $\begin{array}{c}\text { Percentual de unidades } \\
\text { habitacionais adaptadas }\end{array}$ \\
\hline Hotel & $8,4 \%$ \\
Resort & $0,1 \%$ \\
Hotel Fazenda & $0,1 \%$ \\
Pousada & $0,7 \%$ \\
Flat/Apart-hotel & $0,2 \%$ \\
\hline Total & $\mathbf{9 , 5 \%}$ \\
\hline
\end{tabular}

Fonte: elaborada pelos autores (2016).

Calculamos que, das 18.361 unidades habitacionais registradas em SC, apenas 9,5\% possuem algum tipo de adaptação para acessibilidade (Figura 5). 
Figura 5 - Percentual de UH com adaptações para portadores de necessidades especiais em SC.

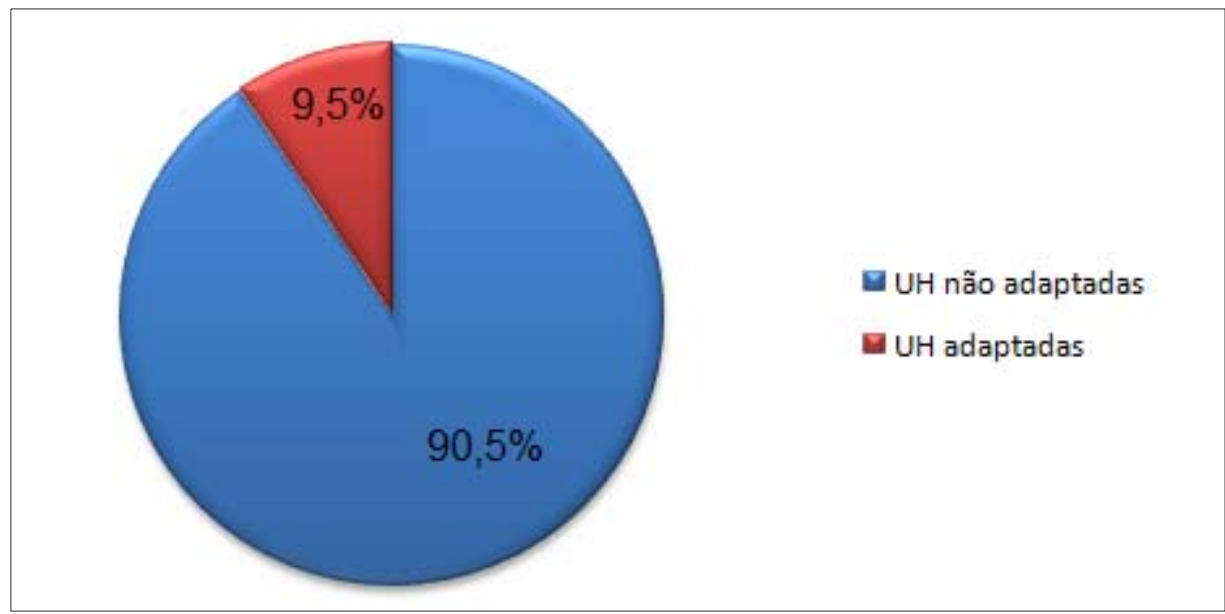

Fonte: elaborada pelos autores (2016).

Do total de unidades habitacionais adaptadas, a maioria das adaptações realizadas visam pessoas cadeirantes, tanto em hotéis quanto em pousadas. No que se refere à adaptação às pessoas com deficiências auditivas, estas aparecem em menor quantidade, em ambos os estabelecimentos. (Figuras 6 e 7).

Figura 6 - Distribuição dos tipos de adaptações

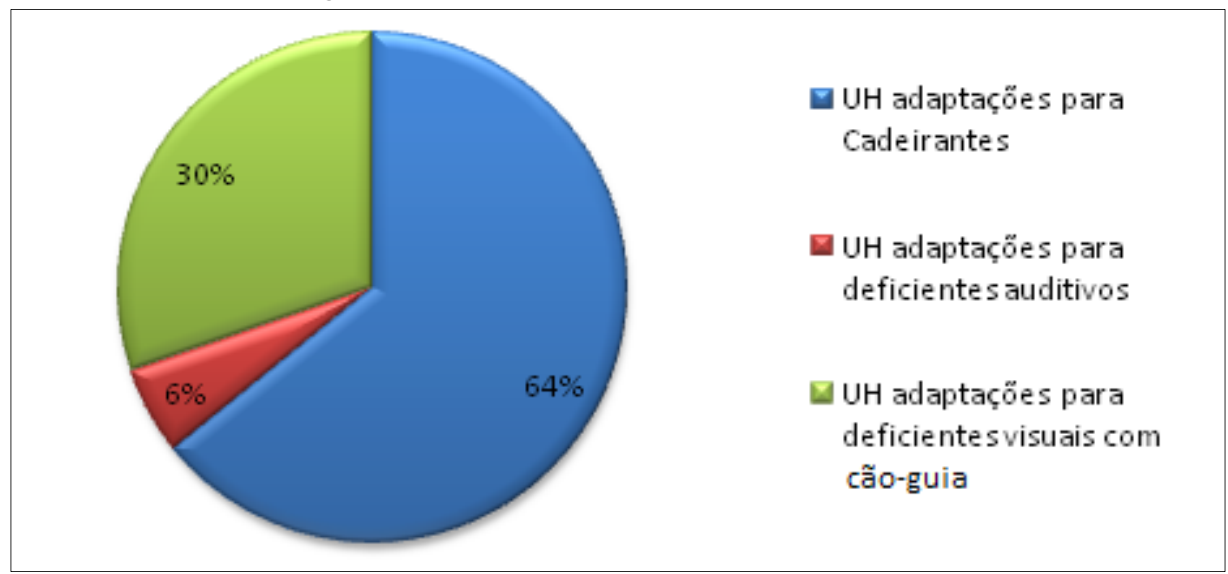

Fonte: elaborada pelos autores (2016).

Figura 7 - Distribuição dos tipos de adaptações para acessibilidade em UH de pousadas em SC

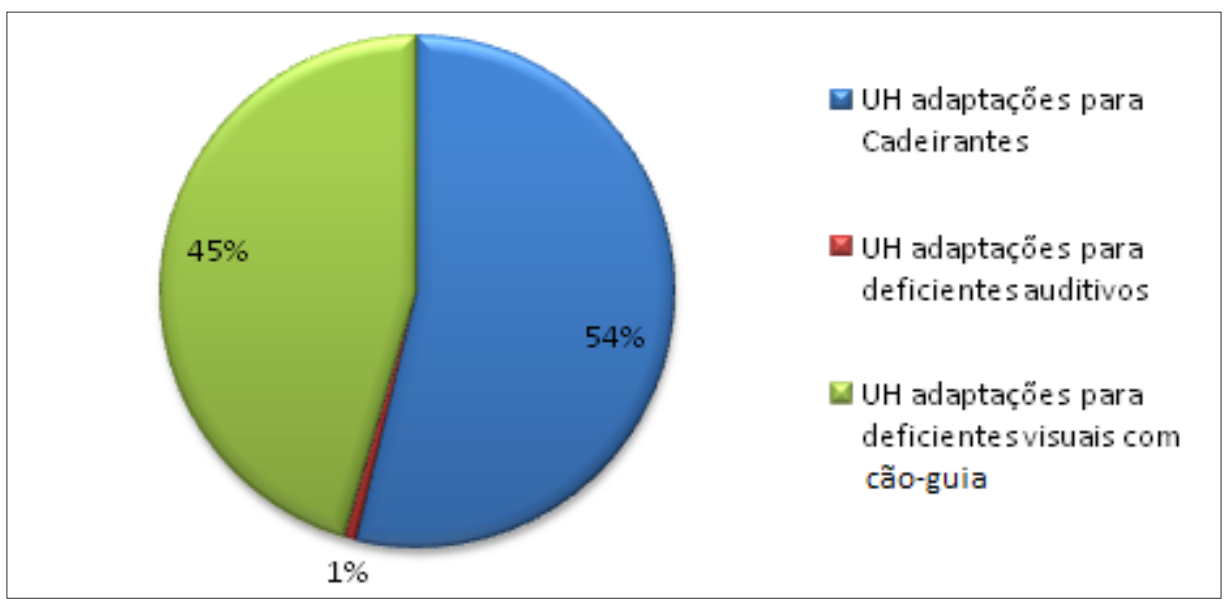

Fonte: elaborada pelos autores. 


\section{CONCLUSÃO}

É reconhecida a importância de Santa Catarina no cenário do turismo brasileiro, principalmente no que se refere ao turismo de lazer. Com a crescente demanda por serviços que atendam também às pessoas com deficiência espera-se que este seja um nicho do mercado a ser explorado pelos meios de hospedagens da região, a fim de aumentar as suas taxas de ocupação. No entanto, para que isto aconteça, percebemos que são necessários esforços do setor neste sentido.

A pesquisa mostrou que, em geral, a parcela de unidades habitacionais e leitos adaptados para atender a este público-alvo é pequena, quando relativa ao total existente. Menos de $10 \%$ das unidades habitacionais são adaptadas. Se levarmos em conta que os meios de hospedagens que fizeram "algum tipo de adaptação", ainda não atenderam todas as exigências da NBR 9050, este percentual seria ainda menor, em um panorama mais realista.

Podemos avaliar também que a parcela que investiu em acessibilidade está basicamente representada pelos hotéis e pousadas, deixando uma lacuna maior quando se trata de outros tipos de meios de hospedagens, como hotéis-fazenda ou apart-hotel/flats, sendo que, a maior parte das adaptações estão focadas nos portadores de necessidades especiais cadeirantes.

Tendo presente este cenário, Santa Catarina ainda precisa investir em adequações para que possa, efetivamente, expandir para o turismo adaptado.

\section{REFERÊNCIAS}

ASSOCIAÇÃO BRASILEIRA DE NORMAS TÉCNICAS (ABNT). NBR 9050: Acessibilidade de pessoas Portadoras de Deficiência a Edificações, Espaços, mobiliário e Equipamentos Urbanos. Rio de Janeiro, 2004.

BRASIL. Decreto n 5.296, de 2 de dezembro de 2004. Regulamenta as Leis nos 10.048, de 8 de novembro de 2000 e 10.098, de 19 de dezembro de 2000. [2004]. Disponível em: < http://www.planalto.gov.br/ccivil_03/_ato2004-2006/2004/decreto/d5296.htm>. Acesso em: 26 out. 2016.

ELY, V. H. M. B.; SILVA, C. S. Unidades habitacionais hoteleiras na llha de Santa Catarina: um estudo sobre acessibilidade espacial. Produção, São Paulo, v. 19, n. 3, p. 489-501, 2009. Disponível em: $<$ http://www.scielo.br/scielo.php? script=sci_arttext\&pid=S0103-65132009000300007\&lng=en\&nrm=iso >. Acesso em: 11 jul. 2016.

EMBRATUR. Manual de Recepção e Acessibilidade de Pessoas Portadoras de Deficiência a Empreendimentos e Equipamentos Turísticos (MRAPPDEET). Brasília: EMBRATUR, 1997.

FECOMÉRCIO. Cresce mercado de turismo adaptado para pessoas com deficiência. [2016]. Disponível em: <www.fecomercio.com.br/noticia/cresce-mercado-de-turismo-adaptado-parapessoas-com-deficiencia>. Acesso em: 1 abr. 2016.

FUSTER, L. F. Teoria e técnica do turismo. Campinas: Papirus, 1999.

INSTITUTO BRASILEIRO DE GEOGRAFIA E ESTATÍSTICA (IBGE). Pesquisa de serviços e

hospedagens. [2011]. Disponível em: 
<http://www.ibge.gov.br/home/estatistica/economia/comercioeservico/psh/2011_todas_regioes/ defaulttabzip.shtm>. Acesso em: 3 abr. 2016.

ORGANIZAÇÃO DAS NAÇÕES UNIDAS (ONU). A ONU e as pessoas com deficiência. [2016].

Disponível em: <https://nacoesunidas.org/acao/pessoas-com-deficiencia/> Acesso em: 3 jun. 2016.

RUSCHMANN, Doris. Marketing Turístico. Campinas, SP: Papirus, 2001.

TRIGO, Luiz Gonzaga Godoi. Turismo básico. 3. ed. São Paulo: SENAC, 1999. 